\title{
Scholarly Design?
}

Marjan Eggermont and Colin McDonald

Schulich School of Engineering, University of Calgary I Special Snowflake Inc.

meggermo@ucalgary.ca I colin@specialsnowflake.ca

\begin{abstract}
Upon entering the School of Engineering from the practice-based Faculty of Fine Arts questions arose regarding the pedagogy of design theory versus the design work of first year engineering students. Fine Arts, in the past couple of years, has tried to enter higher levels of academia by starting to offer PhD programs that are practice-based. Often times these degrees have an aspect of theorizing practice into analysis and thereby equate the process of creating art to the process of scientific experimentation. If one looks at the area of scientific inquiry, which is according to Heidegger: "nothing less than the
\end{abstract}

making secure of methodology over what ever is (nature and history)', questions arise over whether theorizing practice into analysis is the right approach. By doing the latter, one calls into question whether practice by itself is valid and whether by creating a (somewhat) artificial analysis one diminishes the process of practice to a secondary activity.

This paper will start by looking at research done in the area of Fine Arts to solve the 'theorizing practice into analysis' question by creating a situation where practice can be considered as more than mere experimentation. In addition there will be a discussion as to how these findings might be applied to Engineering Design.

\section{Introduction}

'...the implication for engineering design education is a move towards praxis rather than theoria or techne.'

Much research has been done in the past ten years in the Arts, which called into question the acceptance of practice versus the acceptance of research. Many practice-based areas had been brought into universities often for political reasons (amalgamating institutions and streamlining student access). Fine Arts is now in a situation where it has to start offering PhD programs for art students to compete in the world of academic grants and for artists to be allowed to teach incoming students.

Many are still questioning whether this necessarily creates better artists or just better academics (which is necessary if one wants to be considered for research grants). There is an overlap with Engineering in this regard in that apprenticeship opportunities have been lost, but academic requirements have steadily increased.

Many Fine Arts classes are still largely conducted as an apprenticeship. In engineering education these situations are now somewhat present in our relatively new first year design and communication and fourth year capstone design courses.
The paper will look at two extremes in terms of design paradigm:

- A rational problem-solving paradigm

- A reflective action paradigm

The post-positivism model of Karl Popper will be discussed as one of the potential bridging mechanisms between the two design approaches mentioned above.

The next section looks at the seven levels of design expertise identified by Hubert Dreyfus. Dreyfus, an American philosopher, distinguishes seven levels of expertise, corresponding with seven ways of perceiving, interpreting, structuring and solving problems.

\section{Practice as research}

Fine Arts faculties across Europe, Australia, and now Canada have started the process of creating $\mathrm{PhD}$ programs. The Europeans specifically have created the two-tiered system consisting of research-on-practice (science-based) and practice-asresearch (traditional arts based):

1. Research-into-practice (or theorizing practice into analysis) results in a body of artistic work and a systematic analysis of the research findings pertaining either to the art itself or to the 
process of performing or creating it. Candidates must demonstrate a coherent body of artistic work as well as a diversified approach to their professional practice. Their work might be a comprehensive study of various facets (historical, sociological, philosophical, compositional, technical) of a carefully delimited topic.

2. Practice-as-research positions the research itself as a systematic multidisciplinary or interdisciplinary creative process in which the artistic product, supplemented by the research documentation, forms the result of the study. This involves an expansion or renewal of the artistic vocabulary - a search for new forms of viable artistic expression [docArtes].

The latter category could be explored in a program including 'engineering design' that requires the students to maintain a certain amount of 'macro thought' - the ability to compartmentalize their thinking processes in such a way that they can learn from trial-and-error while understanding the real application of abstract design theories. For example, while a prototype produced in a design class (first-year or capstone) can serve as a signifier of the 'goodness' of the design process; it can show how well a student team was able to apply abstract theories to specific problems. Further, the practiceas-research tier could bridge between engineering design methodology and engineering design application, where the actual design object, prototype or product becomes of greater importance. The assessment of the 'outcome' could become an important aspect of judging the practicality of engineering design education.

\section{Engineering design dualities, positivism, and phenomenology}

While engineering teaching and scholarship have remained closely connected with the academic disciplines of science they have remained largely isolated from the inescapable pragmatics of engineering professional practice - the meaning of, and social impact of artifacts leading from engineering design. This larger issue is a product of a number of dualities that currently exist in engineering design education as remnants of the post-Grinter report era. We will attend to two examples: one duality relates to the loss of context and the social captivity of engineering; another duality is exposed in the design task itself.

\section{1 logical positivism versus corporate stakeholders in design}

Engineering tends to be viewed as the application of the natural sciences and logical positivism. More radical Logical Positivists, such as A. J. Ayer, argue that all assertions about moral, aesthetic and religious values are scientifically unverifiable and therefore neither true nor false, but simply meaningless. Since 'all the principles of scientific practice assumed to cover those of engineering as well' there can be no other motivator for the engineered object other than pure objective functionality.

Scientific method, the structure of hypothesis, proof, validation, publication, and critique, is clearly embedded in the scientific culture and serves it well. However, much of this academic endeavor has been essentially de-contextualized for engineering students. The culture and discourse of science impose their own rigor on the academic discipline of engineering and when adopted by engineering, their authority becomes almost absolute. Practitioners commonly believe themselves immune from the influence of theory or philosophy, but the discipline of engineering remains to a great extent captive to the sorts of ideas developed within positivism, due to the overwhelming dominance exercised by positivism in the development of scientific thought during this century. The irony of this situation is that most engineers have probably never heard of Logical Positivism or any of its philosophical spokespersons. As Keynes (1936) noted in a parallel context: 'Practical men, who believe themselves to be quite exempt from any intellectual influences, are usually the slaves of some defunct economist.' Such influences, often exercised almost subliminally, can add up to real cultural bondage' [3].

Simultaneously though, in '[practice] the context of engineering is being framed and governed by corporate managerial hierarchies rather than by the engineering profession itself' [4]. The result is that engineers tend to identify with the interests of their employers (and their stakeholders) and tend not to, as the 'oath of the engineer' suggests, protect the public interest through functionality. Instead the corporate engineering designer tends to drive design through 'profitability'.

\section{2 positivistic epistemology versus phenomenological reflective design}

Within engineering design itself the task of problem solving exposes another duality. Kees Dorst, in his 
article On the Problem of Design Problems [5], identifies these two ends of the design problem-solving spectrum:

- A rational problem-solving paradigm based on positivistic epistemology

- A reflective action paradigm that is largely phenomenological in nature

As outlined above, the main feature of positivism is an insistence on a scientific approach to the human and natural world: a tendency to organize and classify, in particular the developmental stages of the sciences and of human thought in general [6]. Positivism claims that a person lives in an objective world, which can be known through his/her senses; the sensory data are structured by an internal processing system. To know the objective world, a person should study it carefully and dispassionately, preferably with scientific methods.

The reflective action paradigm on the other hand recognizes that a person a dynamic, emotive social being with a history and an environment that heavily influences the person's construction of reality: technology affects our experiences in ways that are not bound to questions of functionality. The discipline of phenomenology may be defined initially as the study of structures of experience, or consciousness. Literally, phenomenology is the study of 'phenomena': the appearance of things, or things as they appear in the subjective or first person point of view - thus the meanings 'things' have in our experience.

Phenomenology (the study of our experience of things) is a potential tool here. It is distinguished from, but related to, the other main fields of philosophy such as ontology (the study of being or what is), epistemology (the study of knowledge), logic (the study of valid reasoning), ethics (the study of right and wrong action), and so on. Phenomenology as a philosophical tradition was launched in the first half of the 2oth century by Edmund Husserl, Martin Heidegger, Maurice Merleau-Ponty, Jean-Paul Sartre, et al, and was prized as the proper foundation of all philosophy - as opposed, say, to ethics or metaphysics or epistemology. The methods and characterization of the discipline were widely debated by Husserl and his successors, and these debates continue to the present day. (The definition of phenomenology offered above will thus be debatable, for example, by Heideggerians, but it remains the starting point in characterizing the discipline.) Recently, the term 'phenomenology' has often been restricted to the characterization of sensory qualities of seeing, hearing, etc.: what it is like to have sensations of various kinds. However, our experience is normally much richer in content than mere sensation - tradition phenomenology is given a much wider range in addressing the meaning things have in our experience, notably as these things arise and are experienced in our 'life-world': the significance of objects, events, tools, the flow of time, the self, and others.

As such reflective action assumes the design process is influenced by all these factors.

\section{Post-positivism}

'Engineers have accepted inappropriate constraints placed upon their profession by the discourse of commerce and science, which have been permitted to define and delimit what engineering is and can be. The result has been a serious limitation in engineers' capacity to examine the social meanings and effects of their work and to self-consciously reflect upon their practice and professional identity'[7].

Post-positivism is a rejection of the central tenets of positivism. While most common forms of postpositivism also posit that there is an independent reality that science can study, it recognizes that all observation is fallible and that all theory is potentially revisable - we cannot know this independent reality with certainty. Further, post-positivism holds that all observations are inherently theoryladen and that we are inherently biased by our cultural experiences, world views, and so on. Once said though, post-positivism rejects the idea that our different experiences and cultures ensure confusion: though our perceptions and observations are fallible, and the resultant constructions are imperfect, we can achieve objectivity by 'triangulating' across these multiple fallible perspectives. Thus, objectivity is an inherently social phenomenon. For example, the process of peer criticism of work will eventually approach objectivity [8].

The post-positivism of Karl Popper's model can encapsulate these seemingly conflicting models along with their intrinsic dualities. Popper identifies three worlds an engineering designer should be able to address and integrate:

1. The theory world: the world of engineering theory and design methods analogous to the current positivism of engineering design academia.

2. A subjective internal world: each individual's world of designing analogous to Dorst's reflective action paradigm and phenomenological reflective design. 
3. An objective external world: the public observed consequences of engineering

In the next section we will look at seven levels of design expertise identified by Hubert Dreyfus in 2003. These levels could be a potential bridge between the two paradigms discussed above in addition to or expanding upon the Popper model.

\section{Levels of design expertise}

A major advantage of accepting that every designer operates within an amalgam of these three worlds (the theory world, the subjective internal world, and the objective external world), is that we can identify levels of design expertise through each of the three. Hubert Dreyfus, an American philosopher, distinguishes seven levels of expertise, corresponding with seven ways of perceiving, interpreting, structuring and solving problems (i.e., the three worlds):

1. Novice: A novice will consider the objective features of a situation, as they are given by the experts, and will follow strict rules to deal with the problem.

2. Advanced Beginner: For an advanced beginner the situational aspects are important, there is a sensitivity to exceptions to the 'hard' rules of the novice. Maxims are used for guidance through the problem situation.

3. Competent: A competent problem solver works in a radically different way. He/she selects the elements in a situation that are relevant, and chooses a plan to achieve the goals. This selection and choice can only be made on the basis of a much higher involvement in the design situation than displayed by a novice or an advanced beginner. Problem solving at this level involves the seeking of opportunities, and of building up expectations. At this level of involvement the problem solving process takes on a trial and error character, and there is a clear need for learning and reflection, that was absent in the novice and the beginner.

4. Proficient: A problem solver that then moves on to be proficient immediately sees the most important issues and appropriate plan, and then reasons out what to do.

5. Expert: The real expert responds to a situation intuitively, and performs the appropriate action straightaway. There is no problem solving and reasoning that can be distinguished at this level of working. This is actually a very comfortable level to be functioning on, and a lot of professionals do not progress beyond this point.

6. Master: With the next level, the master, a new uneasiness creeps in. The master sees the standard ways of working that experienced professionals use not as natural but as contingent. A master displays a deeper involvement into the professional field as a whole, dwelling on success and failure. This attitude requires an acute sense of context, and openness to subtle cues. In his/ her own work the master will perform more nuanced appropriate actions than the expert.

7. Visionary: The world discloser or 'visionary' consciously strives to extend the domain in which he/she works. The world discloser develops new ways things could be, defines the issues, opens new worlds and creates new domains. To do this a world discloser operates more on the margins of a domain, paying attention to other domains as well, and to anomalies and marginal practices that hold promises for a new vision of the domain. [9]

These seven levels can be shown graphically as a set of overlays and intersections of three shapes that could correspond to the three Popper worlds (figure 1): the silhouette of the shapes convey a measure of the elegance of the design process, while the inner shapes display the relative complexity of the interaction between worlds.

Moving from (1) to (5) tends toward the increasingly complex silhouette, while (6) and (7) display a move toward a less dissonant design process. Conversely, the inner shapes generally increase in complexity toward (7), the visionary, as the interrelations between the worlds increase.

\section{Conclusion}

The dualities inherent in engineering design are largely due to academic positivism opposing phenomenology-based practice. By choosing to move to a post-positivist outlook we can encapsulate the two world-views into one design approach.

Further, we could use concepts from Dreyfus to begin to categorize how designers mature and function without creating new dualities between world-views. 


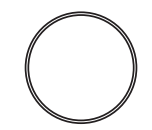

1. Novice
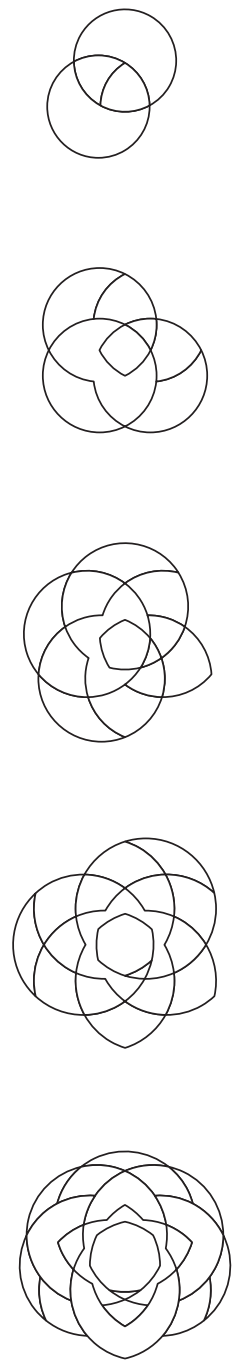

5. Expert

\section{Competant}

4. Proficient

6. Master

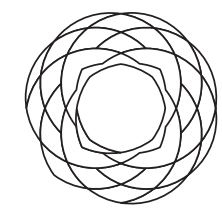

\section{References}

[1]. Terence Love - paper, p. 6

[2]. Marshall, T. \& S. Newton (2000) Scholarly design as a paradigm for practice-based research Working

Papers in Art and Design 1 Retrieved 12 June 2007 from URL http://www.herts.ac.uk/artdes/research/papers/ wpades/voli/marshall2.html ISSN 1466-4917)

[3]. S. Johnston, A. Lee, and H. McGregor, Engineering as captive discourse, Society for Philosophy and Technology, Spring 1996, Vol. 1, Numbers 3-4, p. 2.

[4]. quote section 5: S. Johnston, A. Lee, and H. McGregor, Engineering as captive discourse, Society for Philosophy and Technology, Spring 1996, Vol. 1, Numbers 3-4, p. 2. [5]. K. Dorst, On the problem of design problems: problem solving and design expertise, The journal of design research, 2004, vol 4, issue 2.

[6]. http://www.philosophyprofessor.com/philosophies/ positivism.php | accessed 12 June 2007

[7]. quote section 5: S. Johnston, A. Lee, and H. McGregor, Engineering as captive discourse, Society for Philosophy and Technology, Spring 1996, Vol. 1, Numbers 3-4, p. 1.

[8]. http://www.socialresearchmethods.net/kb/positvsm. php I accessed 12 June 2007

[9]. K. Dorst, I. Reymen, Levels of expertise in design education, proceedeings from the International engineering and product design education conference, September 2004.

\section{Figure 1: levels of design expertise.}

